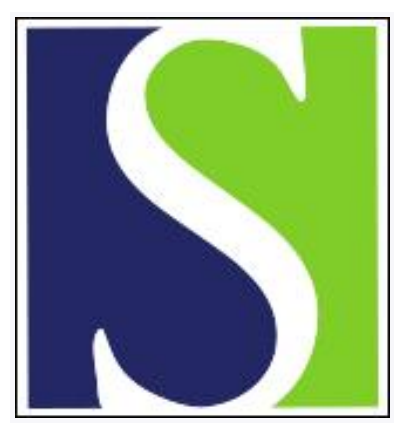

Scand J Work Environ Health 1986;12(4):417-419

https://doi.org/10.5271/sjweh.2120

Issue date: Aug 1986

Physiological noise and its influence on vibrotactile perception thresholds.

by Piercy JE, Brammer AJ, Taylor W

This article in PubMed: www.ncbi.nlm.nih.gov/pubmed/3775332

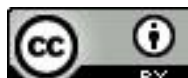




\title{
Physiological noise and its influence on vibrotactile perception thresholds
}

\author{
by Joseph E Piercy, PhD, ${ }^{1}$ Anthony J Brammer, $\mathrm{PhD},{ }^{1}$ William Taylor, $\mathrm{DSc}{ }^{2}$
}

\begin{abstract}
PIERCY JE, BRAMMER AJ, TAYLOR W. Physiological noise and its influence on vibrotactile perception thresholds. Scand $J$ Work Environ Health 12 (1986) 417-419. Vibration of physiological origin (physiological noise) was studied when a small diameter probe, attached to a vibrator and accelerometer for the determination of vibrotactile perception thresholds, was held in contact with a fingertip. The acceleration spectrum consisted of a broad plateau between 0.1 and $10 \mathrm{~Hz}$, where the power spectral density was about $-20 \mathrm{~dB}$ re $1\left(\mathrm{~m} / \mathrm{s}^{2}\right)^{2} / \mathrm{Hz}$, and it fell rapidly with increasing frequency above $10 \mathrm{~Hz}$. Substantial contributions from respiration $(0.2-1 \mathrm{~Hz})$, blood circulation $(1-5 \mathrm{~Hz})$, and hand tremor $(6-8 \mathrm{~Hz})$ could be identified. The physiological noise was largely independent of subject, contact force, and probe diameter, and it was approximately equal in amplitude to the threshold of the vibrotactile perception. Current knowledge of the masking and adaptation of vibrotactile signals indicates that vibrotactile thresholds in the frequency range $2-250 \mathrm{~Hz}$ may be influenced by the magnitude of this physiological noise under some conditions of flesh stimulation.
\end{abstract}

Key terms: hand-arm vibration, objective tests, pacinian corpuscles, tremor, vibration syndrome.

Vibrotactile perception has recently been suggested as a measure of the extent of sensory loss in many disorders affecting the peripheral nerves (2). The success of this method for detecting neurological changes in the hands of workers who repeatedly operate vibrating hand-held power tools has, however, been limited. In an attempt to establish possible reasons for these conflicting clinical results, the physical basis of the method of measurement has been reexamined. In so doing, it has become apparent that a fundamental mechanism has been overlooked, namely, the excitation of vibration of physiological origin when the probe used to determine the perception threshold is brought into contact with the skin. By analogy with the corresponding noise source occurring in the measurement of hearing thresholds (6), this vibration will be called physiological noise. In audiometry (5) the hearing threshold is determined, in principle, by the detection of a signal against a background of this self-noise.

In this paper, which contains our initial findings, the role of physiological noise in vibrotactile perception is explored. Thus, what is the movement of a flattopped cylindrical probe in contact with a fingertip? How does it depend on the range of parameters com-

\footnotetext{
1 Division of Physics, National Research Council of Canada, Ottawa, Ontario, Canada.

2 Department of Community Medicine, University of Dundee, Dundee, Scotland.
}

Reprint requests to: Dr JE Piercy, Division of Physics, National Research Council of Canada, Montreal Road, Ottawa, Ontario K1A OR6, Canada. monly used for measuring vibrotactile thresholds? And what is its relationship with the thresholds observed?

\section{Experimental procedure}

\section{Measurement of thresholds}

The threshold of vibrotactile perception at a fingertip was measured according to the configuration shown as an inset in figure 1, which simulates common clinical practice. A hand was placed palm down on a flat, horizontal surface with the forearm unrestrained and resting on a horizontal, cushioned arm rest. The finger was supported in a natural position at the first joint. A cylindrical, flat-topped probe was attached directly to a Bruel \& Kjaer (B \& K) accelerometer (type 4333), which was mounted on the vibrating table of a minivibrator (B \& K model 4810). The driver was mounted in turn on a beam balance so that the probe could be held in contact with the fingertip by a known, steady, vertical force. The amplitude sensitivity of this measuring system, together with its associated electronics, was checked with an accelerometer calibrator, and the reference levels quoted are zero-to-peak amplitudes.

With this apparatus the threshold of perception was determined with the elementary psychoacoustic techniques of manual audiometry. The stimulus consisted of pulsed pure tones in the frequency range of $2-250$ $\mathrm{Hz}$, beginning and ending at a zero crossing. Their harmonic distortion was low (typically $\sim-30 \mathrm{~dB}$ re the fundamental). The duration of the tone bursts for frequencies $\mathrm{f}>10 \mathrm{~Hz}$ was typically $\mathrm{l} \mathrm{s}$, and, for $\mathrm{f}<10$ $\mathrm{Hz}$, it was the time corresponding to 10 cycles of oscillation. Measurements were conducted on the index or second finger when the temperature of the fingertip was in the range $28-33^{\circ} \mathrm{C}$. 


\section{Measurement of physiological noise}

The same apparatus was used to measure the physiological noise of a fingertip after the electrical input to the minivibrator was switched off. Data were obtained from either the index or second fingers of three adult male subjects for different probe diameters and contact forces. At all frequencies the physiological noise exceeded the residual noise of the measuring system by at least $20 \mathrm{~dB}$.

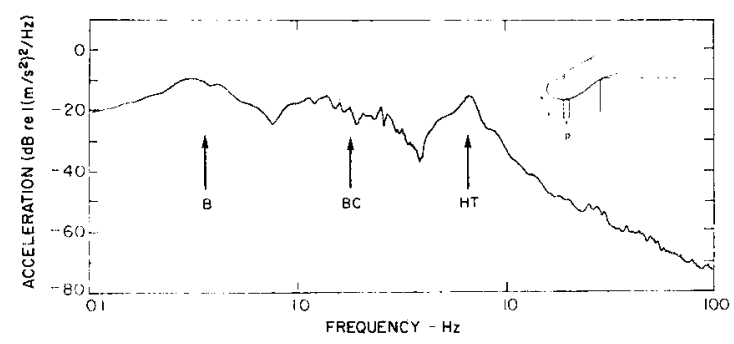

Figure 1. Power spectral density of physiological noise, measured as acceleration, for one subject. The inset shows the measurement configuration. $(p=$ probe, $B=$ breathing, $B C=$ blood circulation, $H T=$ hand tremor)

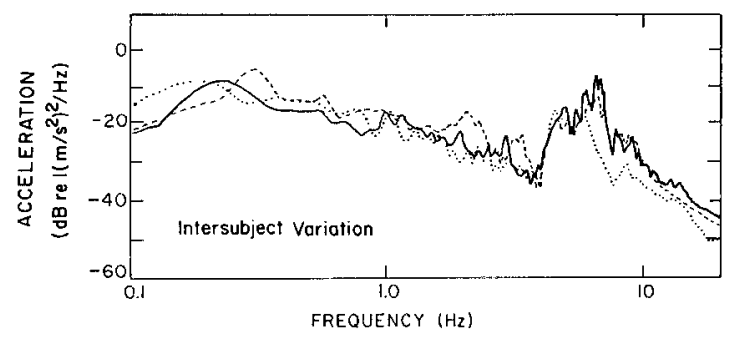

Figure 2. Power spectral density of physiological noise for three male subjects aged 30 to 60 years.

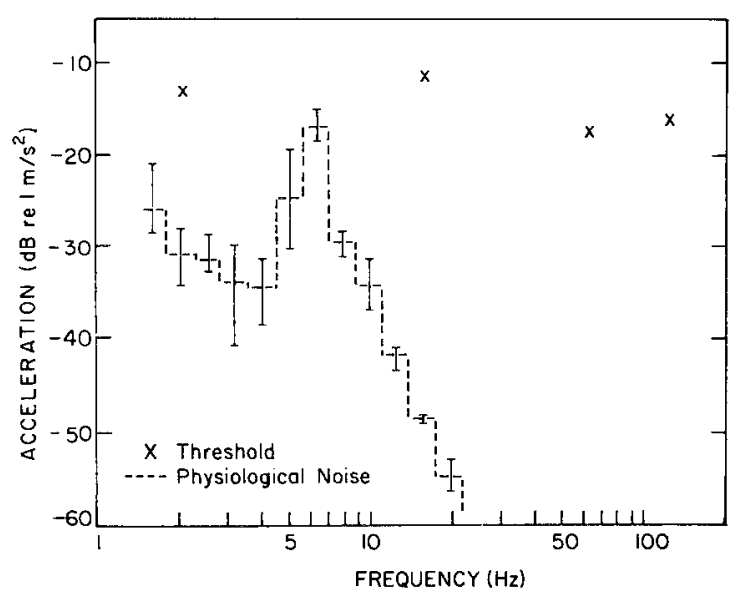

Figure 3. Vibrotactile perception thresholds and physiological noise measured simultaneously in one-third octave bands on one subject.

\section{Results and discussion}

The spectrum of the physiological noise observed at the fingertip of a 30-year-old male is shown in figure 1. It was obtained with a fast Fourier transform frequency analyzer, using a filter bandwidth of 0.05 $\mathrm{Hz}$, and an exposure time of $3 \mathrm{~min}$ hence had a standard statistical error of $1.5 \mathrm{~dB}$. A probe diameter of $3 \mathrm{~mm}$ was used and a contact force of $0.2 \mathrm{~N}$. Note that the spectral density in the frequency range $0.1-8$ $\mathrm{Hz}$ remained generally within $10 \mathrm{~dB}$ of $-20 \mathrm{~dB}$ re 1 $\left(\mathrm{m} / \mathrm{s}^{2}\right)^{2} / \mathrm{Hz}$. As the frequency increased above $10 \mathrm{~Hz}$, the level dropped steadily to about $-80 \mathrm{~dB}$ at $100 \mathrm{~Hz}$ (and remained at or below that level to $1 \mathrm{kHz}$ although not shown in this presentation). The coincidence of the broad peak at low frequencies with the measured frequency of breathing $(0.3 \mathrm{~Hz})$ suggests a substantial contribution to physiological noise from breathing in the frequency range of $0.1-1.0 \mathrm{~Hz}$. Several peaks also occurred in the frequency range of $1-5 \mathrm{~Hz}$, most of which can usually be identified with the measured pulse frequency $(\sim 1 \mathrm{~Hz})$ and its harmonics. The contributions from breathing and blood circulation corresponded reasonably well to those proposed earlier by Johansson \& Vallbo (3) in their neurophysiological measurements of mechanoreceptor performance.

Throughout the measurements there was usually a broad peak centered at a frequency of $6-8 \mathrm{~Hz}$ (marked HT in figure 1), sometimes with the sharp maximum evident in the figure. The most likely origin of this peak was a substantial contribution from hand tremor, which has been known to have a resonance peak consistently in this frequency range (7). Thus the method of supporting the hand, arm, and finger and the mechanical compliance of the arm rests or supports may be expected to influence the physiological noise at these frequencies.

For a single subject, the repeatability of the spectrum of physiological noise is typically within $\pm 3 \mathrm{~dB}$, ie, comparable to the intersubject variation shown in figure 2. We explored the effect of the coupling between the flesh and the probe tip by varying the contact force and probe diameter over the ranges normally employed in clinical studies, namely, forces from 0.02 to $0.2 \mathrm{~N}$ and probe diameters from 3 to $10 \mathrm{~mm}$. (Since contact with the flesh of the fingertip does not expand significantly beyond a diameter of $10 \mathrm{~mm}$, the larger probes sometimes employed will not further vary the coupling.) Changes in the spectra of physiological noise with this variation in parameters were small, comparable with the inter- and intrasubject variability.

Simultaneous measurements of physiological noise and pure-tone thresholds at a fingertip are shown in figure 3 . The broken line gives the average one-third octave-band spectrum of physiological noise during threshold determinations at four frequencies, and the error bars show the maximum deviations that occurred from run to run. Within the limited accuracy, estimated to be $\pm 5 \mathrm{~dB}$ and set mainly by the psycho- 
physical method, figure 3 indicates that the perception threshold is independent of frequency (ie, constant acceleration within this frequency range) and equal to the peak level of physiological noise. Results similar to those shown for one subject in figure 3 were obtained from the three subjects.

In view of the preliminary nature of the reported threshold determinations, a comparison is made with more established threshold determinations in figure 4. The two sets of triangles show the measurements on the subject for figure 3 from two different days. A line of constant acceleration (the solid line), which is characteristic for the response of pacinian corpuscles in this region of frequencies (8), has been drawn as an approximate fit to this data.

It is evident from figure 4 that the results of all three studies are in good agreement at frequencies above 30 $\mathrm{Hz}$. At these frequencies it is generally accepted that the pacinian corpuscles are responsible for the threshold of vibrotactile perception (8). At frequencies below $30 \mathrm{~Hz}$, the results of Mountcastle et al (4) and Gescheider et al (1) fall below the constant acceleration line, through stimulation of more sensitive mechanoreceptors. In the present measurements the thresholds were apparently determined by the pacinian corpuscles at all frequencies, because of somewhat different experimental parameters. (The differences below $30 \mathrm{~Hz}$ will be pursued elsewhere.)

Now Verrillo, Gescheider, and co-workers (8) have established, by a series of carefully controlled psychophysical experiments, that a masking or adapting stimulus uniformly raises pure-tone vibrotactile perception thresholds across the frequency band of a given mechanoreceptor approximately up to the level of that stimulus.

In consequence, the results shown in figure 3 imply that the new measurements of vibrotactile thresholds for the pacinian system reported in this presentation, including those indicated by the triangles in figure 4 , are likely determined by the level of physiological noise, either directly through masking or through adaptation.

The role of physiological noise in previous determinations of vibrotactile thresholds is much more problematic, of course, because of the lack of concurrent noise measurements. Moreover there will be some dependence of the noise levels on unexplored variables, such as the mechanical impedance of the driver. Nevertheless, the levels of physiological noise have been found to be generally insensitive to experimental factors, such as changes of subject, time, probe diameter, and contact force, and the new thresholds for the pacinian system are in general agreement with some of the better determinations available in the literature (figure 4). A more general role for physiological noise

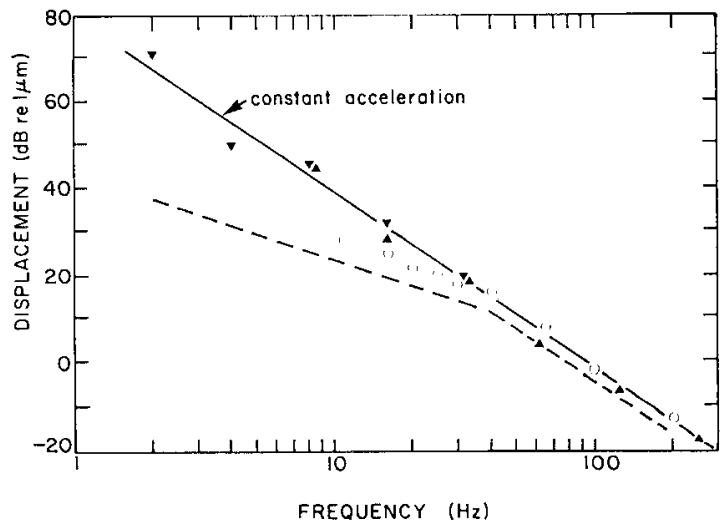

Figure 4. Comparison of measurements of vibrotactile thresholds for one subject (triangles) with those measured previously. The average threshold obtained by Mountcastle et al (4) with seven subjects is shown by the broken line, while those found by Gescheider et al (1) for five subjects are shown by the open circles.

in influencing vibrotactile thresholds is therefore a distinct possibility.

Hitherto the determination of thresholds of vibrotactile perception does not seem to have been considered seriously as the detection of a signal against a background of self-noise, as in audiometry. If vibrotactile measurements are to be developed as a reliable test for neurological dysfunction in the hands, this change in philosophy would seem to be needed.

\section{References}

1. Gescheider GA, Capraro AJ, Frisina RD, Hamer RD, Verrillo RT. The effects of a surround on vibro-tactile thresholds. Sens Process 2 (1978) 99-115.

2. Goldberg JM, Lindblom U. Standardised method of determining vibratory perception thresholds for diagnosis and screening in neurological investigation. $J$ Neurol Neurosurg Psychiatry 42 (1979) 793-803.

3. Johansson RS, Vallbo ÅB. Detection of tactile stimuli: Thresholds of different units related to the psychophysical thresholds in the human hand. J Physiol (London) 297 (1979) 405-422.

4. Mountcastle VB, Lamotte RH, Carli G. Detection thresholds for stimuli in humans and monkeys: Comparison with threshold events in mechano-receptor afferent nerve fibers innervating the monkey hand. J Neurophysiol 35 (1972) $122-136$.

5. Rudmose $\mathrm{W}$. The case of the missing $6 \mathrm{~dB}$. J Acoust Soc Am 71 (1982) 650-659.

6. Shaw EAG, Piercy JE. Audiometry and physiological noise. In: Proceedings of the fourth international congress on acoustics, Copenhagen, 1962, p H52.

7. Stiles RN, Randall JE. Mechanical factors in human tremor frequency. J Appl Physiol 23 (1967) 325-330.

8. Verrillo RT. Psychophysics of vibro-tactile stimulation. J Acoust Soc Am 77 (1982) 225-232. 